\title{
The conserved Lysine69 residue plays a catalytic role in Mycobacterium tuberculosis shikimate dehydrogenase Valnês S Rodrigues Junior ${ }^{1}$, Ardala Breda ${ }^{1,2}$, Diógenes S Santos*1 and Luiz A Basso*1
}

Addresses: ${ }^{1}$ Centro de Pesquisas em Biologia Molecular e Funcional (CPBMF), Instituto Nacional de Ciência e Tecnologia em Tuberculose (INCT-TB), Pontifícia Universidade Católica do Rio Grande do Sul (PUCRS); Porto Alegre, Brazil and ${ }^{2}$ Programa de Pós-Graduação em Biologia Celular e Molecular, Pontifícia Universidade Católica do Rio Grande do Sul (PUCRS); Porto Alegre - RS, Brazil

E-mail: Valnês S Rodrigues Junior - valnesjunior@yahoo.com.br; Ardala Breda - ardalabreda@hotmail.com;

Diógenes S Santos* - diogenes@pucrs.br; Luiz A Basso* - luiz.basso@pucrs.br

${ }^{*}$ Corresponding author

Published: 16 November 2009

BMC Research Notes 2009, 2:227 doi: 10.1186/1756-0500-2-227
Received: 22 May 2009

Accepted: 16 November 2009

This article is available from: http://www.biomedcentral.com/1756-0500/2/227

(C) 2009 Santos et al; licensee BioMed Central Ltd.

This is an Open Access article distributed under the terms of the Creative Commons Attribution License (http://creativecommons.org/licenses/by/2.0), which permits unrestricted use, distribution, and reproduction in any medium, provided the original work is properly cited.

\begin{abstract}
Background: The shikimate pathway is an attractive target for the development of antitubercular agents because it is essential in Mycobacterium tuberculosis, the causative agent of tuberculosis, but absent in humans. M. tuberculosis aroE-encoded shikimate dehydrogenase catalyzes the forth reaction in the shikimate pathway. Structural and functional studies indicate that Lysine69 may be involved in catalysis and/or substrate binding in $M$. tuberculosis shikimate dehydrogenase. Investigation of the kinetic properties of mutant enzymes can bring important insights about the role of amino acid residues for $M$. tuberculosis shikimate dehydrogenase.
\end{abstract}

Findings: We have performed site-directed mutagenesis, steady-state kinetics, equilibrium binding measurements and molecular modeling for both the wild-type $M$. tuberculosis shikimate dehydrogenase and the K69A mutant enzymes. The apparent steady-state kinetic parameters for the $M$. tuberculosis shikimate dehydrogenase were determined; the catalytic constant value for the wild-type enzyme $\left(50 \mathrm{~s}^{-1}\right)$ is 68 -fold larger than that for the mutant K69A $\left(0.73 \mathrm{~s}^{-1}\right)$. There was a modest increase in the Michaelis-Menten constant for DHS $(\mathrm{K} 69 \mathrm{~A}=76 \mu \mathrm{M}$; wild-type $=29 \mu \mathrm{M})$ and NADPH $(\mathrm{K} 69 \mathrm{~A}=30 \mu \mathrm{M}$; wild-type $=1 \mathrm{I} \mu \mathrm{M})$. The equilibrium dissociation constants for wildtype and K69A mutant enzymes are $32( \pm 4) \mu M$ and $134( \pm 21)$, respectively.

Conclusion: Our results show that the residue Lysine69 plays a catalytic role and is not involved in substrate binding for the $M$. tuberculosis shikimate dehydrogenase. These efforts on $M$. tuberculosis shikimate dehydrogenase catalytic mechanism determination should help the rational design of specific inhibitors, aiming at the development of antitubercular drugs.

\section{Background}

Tuberculosis (TB) remains a major global health concern. It has been estimated that one-third of the world population is infected with Mycobacterium tuberculosis, the causative agent of $\mathrm{TB}$, and that 30 million people died from this disease in the last decade [1]. The epidemic of the human immunodeficiency virus, the increase in the homeless population, and the decline in health care structures and national surveillance are contributing factors to $\mathrm{TB}$ resurgence. Inappropriate treatment regimens and patient noncompliance in completing the therapies are associated with the 
emergence of multi-drug resistant TB (MDR-TB), defined as strains of $M$. tuberculosis resistant to at least isoniazid and rifampicin, two pivotal drugs used in the standard treatment of $\mathrm{TB}$ [2]. It has been reported the emergence of extensively drug-resistant (XDR) TB cases, defined as cases in persons with TB whose isolates are MDR as well as resistant to any one of the fluoroquinolone drugs and to at least one of the three injectable second-line drugs [3]. XDR-TB is widespread raising the prospect of virtually incurable TB worldwide [3]. There is thus an urgent need for new drugs to improve the treatment of MDR- and XDR-TB, and to provide more effective drugs to shorten the duration of TB treatment. Enzyme inhibitors make up roughly $25 \%$ of the drugs marketed [4], and are thus important promising drug targets.

The shikimate pathway is an attractive target for the development of herbicides and antimicrobial agents because it is essential in algae, higher plants, bacteria, and fungi, but absent from mammals [5]. The mycobacterial shikimate pathway leads to the biosynthesis of precursors of aromatic amino acids, naphthoquinones, menaquinones, and mycobactins [6], and is essential for M. tuberculosis viability [7]. Shikimate dehydrogenase (SD; EC 1.1.1.25), the fourth enzyme of this pathway, catalyzes the NADPH-dependent reduction of 3-dehydroshikimate (DHS) to shikimate (SHK, Fig. 1). We have previously reported production, characterization, and determination of kinetic and chemical mechanisms of aroE-encoded SD from M. tuberculosis H37Rv strain (MtbSD) [8-10]. Multiple sequence alignment, comparative homology modeling, and $\mathrm{pH}$-rate profiles suggested that the Lysine69 in the DHS/SHK binding site of MtbSD plays a role in substrate binding and/or catalysis [10,11]. Here we describe site-directed mutagenesis, steady-state kinetics, fluorimetric measurements and structural analyses to probe the role of Lys69 in MtbSD and provide insight into the molecular basis of DHS/SHK recognition and/or catalysis.

\section{Methods}

\section{Site-directed mutagenesis}

A previously constructed pET-23a(+)::aroE recombinant vector [8] was used as a template for PCR-based

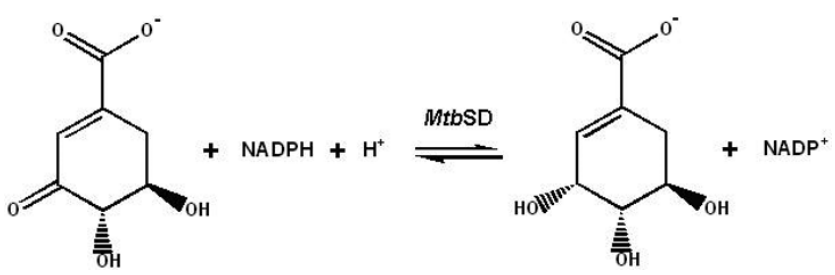

Figure I

The shikimate dehydrogenase-catalyzed reaction. mutagenesis using the Quick Change site-directed mutagenesis kit (Stratagene, La Jolla, CA). The synthetic oligonucleotides employed were as follows: $5^{\prime}$ ggtgtttcggtgaccatgccgggcgcgttcgccgccetgcggttcg-3' (forward) and 5'-cgaaccgcagggcggcgaacgcgccogcatggtcaccgaaacacc- $3^{\prime}$ (reverse) (in bold is the codon for alanine). PfuTurbo ${ }^{\circledR}$ DNA polymerase and standard PCR amplification program were employed. The PCR product was treated with DpnI endonuclease that specifically digests the methylated DNA template, and selects for the mutation-containing synthesized DNA.

\section{Expression, release and purification of recombinant MtbSD}

The recombinant plasmid was introduced into E. coli C41 (DE3) host cells (Novagen, Madison, WI) by electroporation. Single colonies were used to inoculate $2 \mathrm{~L}$ of LB medium containing $50 \mu \mathrm{g} \mathrm{mL}^{-1}$ ampicillin, and $1 \mathrm{mM}$ isopropyl $\beta$-D-thiogalactopyranoside was added to cultures reaching an $\mathrm{OD}_{600}$ of $0.4-0.6$, and grown for $24 \mathrm{~h}$ at $37^{\circ} \mathrm{C}$ at $180 \mathrm{rpm}$. Cells (5 g) were harvested by centrifugation at $14,900 \mathrm{~g}$ for $30 \mathrm{~min}$ at $4^{\circ} \mathrm{C}$, and stored at $-20^{\circ} \mathrm{C}$. The freeze-thaw method was used to release the proteins in the soluble fraction [8]. The purification protocol was as previously described [12]. Samples of the purification steps were analyzed by SDS-PAGE [13] and protein content by the Bradford's method [14].

\section{Enzyme activity assays and determination of kinetic parameters}

Steady-state kinetics measurements of homogeneous MtbSD K69A mutant activity were carried out for the forward direction at $25^{\circ} \mathrm{C}$ in $100 \mathrm{mM}$ potassium phosphate buffer, $\mathrm{pH} 7.3$, by monitoring the decrease in absorbance at $340 \mathrm{~nm}\left(\varepsilon=6220 \mathrm{M}^{-1} \mathrm{~cm}^{-1}\right.$ for $\mathrm{NADPH}$ ) accompanying the conversion of NADPH and DHS to NADP ${ }^{+}$and SHK. The K69A activity was measured at varying final concentrations of DHS (20-300 $\mu \mathrm{M})$ and NADPH at constant saturating level $(200 \mu \mathrm{M})$; and varying NADPH concentrations (20-250 $\mu \mathrm{M})$ and DHS at constant saturating level ( $250 \mu \mathrm{M}$; Fig. $2 \mathrm{~B}$ and $2 \mathrm{D})$. The wild-type SD activity was measured at various DHS concentrations (10-100 $\mu \mathrm{M})$ and NADPH at constant saturating level $(200 \mu \mathrm{M})$; and various NADPH concentrations $(5-50 \mu \mathrm{M})$ and DHS at constant saturating level (250 $\mathrm{\mu M}$; Fig. 2A and 2C). All measurements were in duplicate. Steady-state kinetic constants were obtained by non-linear regression analysis of the kinetic data fitted to the Michaelis-Menten equation $\left(\mathrm{v}=\mathrm{V}_{\max } \times[\mathrm{S}] / \mathrm{K}_{\mathrm{m}}+[\mathrm{S}]\right)$ using the SigmaPlot software (SPSS, Inc).

\section{Fluorescence spectroscopy}

Fluorescence titration was performed in a Shimadzu spectrofluorophotometer RF-5301PC at $25^{\circ} \mathrm{C}$ by making 

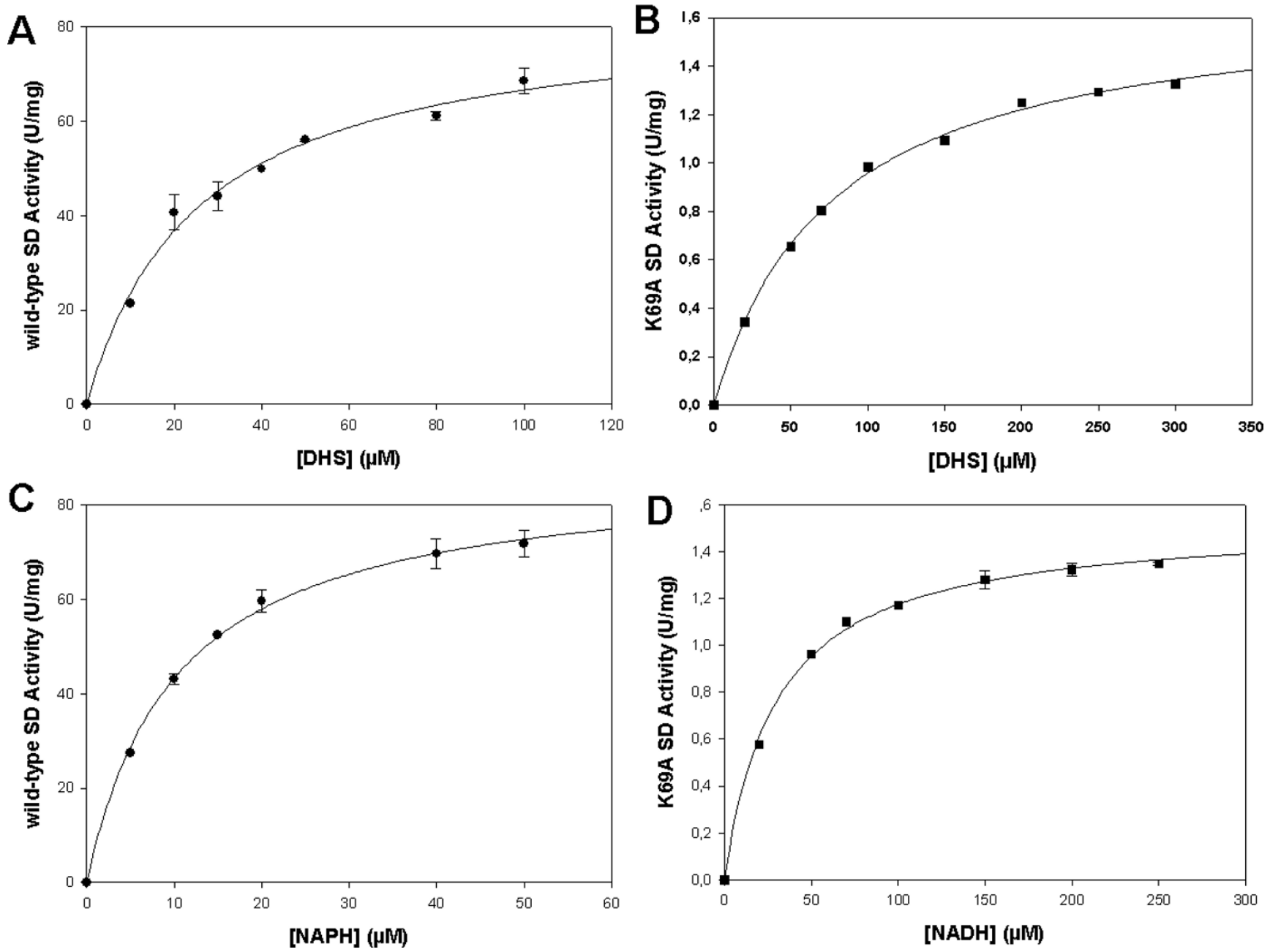

Figure 2

Steady-state kinetic measurements for wild-type (A and C) and K69A (B and D) MtbSD in the forward direction. A and B: DHS concentrations were varied while NADPH concentration was maintained at a fixed saturating level. $\mathrm{C}$ and D: NADPH concentrations were varied while DHS concentration was maintained at a fixed saturating level.

microliter additions of DHS substrate stock solutions to $2 \mathrm{~mL}$ of $1 \mu \mathrm{M}$ of $\mathrm{MtbSD}$ in $100 \mathrm{mM}$ potassium phosphate buffer $\mathrm{pH}$ 7.3. The binding of DHS to MtbSD causes a quench in the intrinsic protein fluorescence $\left(\lambda_{\mathrm{exc}}=300 \mathrm{~nm}\right.$; $310 \leq \lambda_{\text {em }} \leq 450 \mathrm{~nm}$; maximum $\lambda_{\text {em }}$ at $340 \mathrm{~nm}$ ), which allowed monitoring of MtbSD-DHS binary complex formation at equilibrium. To assess the DHS inner-filter effect in the fluorimeter, two cuvettes were placed in series so that the contents of the first cuvette acted as a filter of the excitation light and the light emitted from the second cuvette detected. DHS was added to the first cuvette, while the second cuvette contained the protein. In this manner, DHS inner-filter effect on protein fluorescence could be assessed. The results were plotted to a rectangular hyperbola by using the nonlinear regression function of SigmaPlot 2004 (SPSS, Inc.).

\section{MtbSD K69A structural analysis}

The wild-type MtbSD three-dimensional (3D) model was built previously by comparative homology modeling using Escherichia coli SD as template (PDB ID: 1NYT) [10], however, a more recent 3D structure for Thermus thermophilus SD (PDB ID: 2EV9), solved by X-ray crystallography at $1.90 \AA$ in complex with shikimate [15], was used as a template for MtbSD K69A mutant modeling, as well as to evaluate DHS/SHK substrate binding mode to MtbSD. T. thermophilus SD shows higher primary structure identity to MtbSD ( $30 \%$ identity and $13 \%$ strong similarity) than E. coli SD (26\% identity and $15 \%$ strong similarity), and the presence of both enzyme's cofactor and substrate bound at its active site makes such structure a more suitable template for MtbSD comparative homology modeling. 
Target and template pair-wise sequence alignment required small gaps inclusion on both $M$. tuberculosis and T. thermophilus SD primary sequences. MtbSD K69A model was built by restrained-based homology modeling implemented in MODELLER9v1 [16], with the standard protocol of the comparative protein structure modeling methodology, by satisfaction of spatial restrains [17,18]. Atomic coordinates of SHK heteroatoms were copied from template structure into the MtbSD K69A model. The best model was selected according to MODELLER objective function [19] and subjected to energy minimization for amino acid side chain and main chain rearrangements with GROMACS package [20] using the $43 \mathrm{a} 1$ force-field. The system was submitted to an initial steepest descent energy minimization in vacuo with a maximum number of 400 minimization steps, followed by a maximum of 3000 steps of conjugate gradient energy minimization.

The program PROCHECK and VERIFY 3D were employed to, respectively, analyze stereochemical quality and validate the 3D profile of the model, as previously described [10]. Structural correspondence between MtbSD K69A model and $T$. thermophilus was evaluated by their root-mean square deviation (RMSD). H-bond interactions were evaluated with LIGPLOT v4.4.2 [21], considering an atomic distance cut off of $3.9 \AA$ (program default values).

\section{Findings and Discussion}

\section{Site-directed mutagenesis, protein expression and} purification

Total sequencing of aroE mutant DNA into pET-23a(+) vector confirmed that the mutation was introduced into the expected site and that no unwanted mutations were introduced by the PCR amplification step. The recombinant $M t b S D$ protein purification protocol resulted in a protein yield of $13 \%$, according to the previous protocol [12].

\section{Steady-state kinetic parameters}

The apparent steady-state kinetic parameters for K69A (Fig. 2B and 2D) and wild-type MtbSD (Fig. 2A and 2C) are given in Table 1 . It is noteworthy that the catalytic constant $\left(\mathrm{k}_{\mathrm{cat}}\right)$ value for wild-type $\mathrm{MtbSD}\left(50 \mathrm{~s}^{-1}\right)$ is 68 fold larger than K69A $\left(0.73 \mathrm{~s}^{-1}\right)$, whereas there was a modest increase in the $\mathrm{K}_{\mathrm{m}}$ values for DHS (K69A = $76 \mu \mathrm{M}$; wild-type $\mathrm{MtbSD}=29 \mu \mathrm{M})$ and NADPH $(\mathrm{K} 69 \mathrm{~A}=$ $30 \mu \mathrm{M}$; wild-type $\mathrm{MtbSD}=11 \mu \mathrm{M})$. The apparent secondorder rate constant $\left(\mathrm{k}_{\mathrm{cat}} / \mathrm{K}_{\mathrm{m}}\right)$ values for DHS $(\mathrm{K} 69 \mathrm{~A}=$ $9.6 \times 10^{3} \mathrm{M}^{-1} \mathrm{~s}^{-1}$; wild-type MtbSD $\left.=1.7 \times 10^{6} \mathrm{M}^{-1} \mathrm{~s}^{-1}\right)$ and NADPH $\left(\mathrm{K} 69 \mathrm{~A}=24 \times 10^{3} \mathrm{M}^{-1} \mathrm{~s}^{-1}\right.$; wild-type MtbSD = $\left.4.5 \times 10^{6} \mathrm{M}^{-1} \mathrm{~s}^{-1}\right)$ indicate that the mutant has a lower specificity constant for both substrates. A comparison of $\mathrm{k}_{\mathrm{cat}} / \mathrm{K}_{\mathrm{m}}$ values is an appropriate method to assess the effect(s) of a mutation on substrate(s) binding and catalysis since it includes the activation energies and the
Table I: Apparent steady-state kinetic parameters and equilibrium binding constants for wild type and K69A mutant MtbSD

\begin{tabular}{lcc}
\hline Parameter & Wild-type & K69A \\
\hline $\mathrm{V}_{\max }\left(\mathrm{U} \mathrm{mg}^{-1}\right)^{\mathrm{a}}$ & $110 \pm 2$ & $1.61 \pm 0.03$ \\
$\mathrm{~K}_{\mathrm{m}} \mathrm{DHS}(\mu \mathrm{M})^{\mathrm{a}}$ & $29 \pm 2$ & $76 \pm 4$ \\
$\mathrm{~K}_{\mathrm{m}} \mathrm{NADPH}(\mu \mathrm{M})^{\mathrm{a}}$ & $11.0 \pm 0.6$ & $30 \pm 2$ \\
$\mathrm{k}_{\text {cat }}\left(\mathrm{s}^{-1}\right)^{\mathrm{a}}$ & $50 \pm 1$ & $0.73 \pm 0.0 \mathrm{I}$ \\
$\mathrm{k}_{\text {cat }} / \mathrm{K}_{\mathrm{m}} \mathrm{DHS}\left(\mathrm{M}^{-1} \mathrm{~s}^{-1}\right)^{\mathrm{a}}$ & $1.7( \pm 0.1) \times 10^{6}$ & $9.6( \pm 0.5) \times 10^{3}$ \\
$\mathrm{k}_{\text {cat }} / \mathrm{K}_{m} \mathrm{NADPH}\left(\mathrm{M}^{-1} \mathrm{~s}^{-1}\right)^{\mathrm{a}}$ & $4.5( \pm 0.2) \times 10^{6}$ & $24( \pm 2) \times 10^{3}$ \\
$\mathrm{~K}_{\mathrm{d}} \mathrm{DHS}(\mu \mathrm{M})^{\mathrm{b}}$ & $32 \pm 4$ & $134 \pm 21$ \\
\hline
\end{tabular}

a steady-state kinetic parameters.

${ }^{b}$ spectroscopic measurements of intrinsic protein fluorescence (equilibrium binding).

binding energies [22]. A change of $12.8 \mathrm{~kJ} \mathrm{~mol}^{-1}$ can be calculated when we compare the values of $\mathrm{k}_{\mathrm{cat}} / \mathrm{K}_{\mathrm{m}}$ of DHS reduction for wild-type and K69A MtbSD. These results indicate that the conserved Lys69 residue in $M t b S D$ plays a critical role in catalysis, but plays no role in substrate binding. Moreover, the fact that the K69A MtbSD mutant protein still binds DHS and NADPH with only slightly larger $\mathrm{K}_{\mathrm{m}}$ values as compared to wild-type enzyme indicates that the mutant protein is properly folded giving more confidence that the results are not an artifact generated by the mutation. Linearity of each measurement and dose dependence when adding different volumes of the enzyme solution were confirmed for all enzyme activity assays (data not shown).

\section{Equilibrium binding}

Spectrofluorimetric assays were carried out to determine the equilibrium dissociation constant for MtbSD-DHS binary complex formation (Fig. 3). The change in the intrinsic protein fluorescence at varying DHS concentrations $(2-450 \mu \mathrm{M})$ yielded equilibrium dissociation constant $\left(\mathrm{K}_{\mathrm{d}}\right)$ values of $32( \pm 4) \mu \mathrm{M}$ for wild-type MtbSD and $134( \pm 21) \mu \mathrm{M}$ for K69A mutant (Table 1). Interestingly, measurements of changes in nucleotide fluorescence upon NADPH binding to wild-type MtbSD $\left(\lambda_{\text {exc }}=370 \mathrm{~nm} ; 380 \leq \lambda_{\text {em }} \leq 600 \mathrm{~nm} ;\right.$ maximum $\lambda_{\text {em }}$ at $445 \mathrm{~nm}$ ) did not show any saturation, which indicates a very large $K_{d}$ value. This is consistent with a steady-state ordered bi-bi mechanism with DHS binding first followed by NADPH binding to MtbSD active site [10]. Moreover, these experiments confirmed our proposal that the Lys69 residue plays a minor role, if any, in substrate binding.

\section{Structural analysis}

Two crystallographic structures of SD in complex with both its coenzyme and SHK (Aquifex aeolicus, PDB ID: 2HK9[23] and T. thermophilus, PDB ID: 2EV9[15], solved by X-ray diffraction at $2.20 \AA$ and $1.90 \AA$ resolution, respectively) are available in the Protein Data Bank 


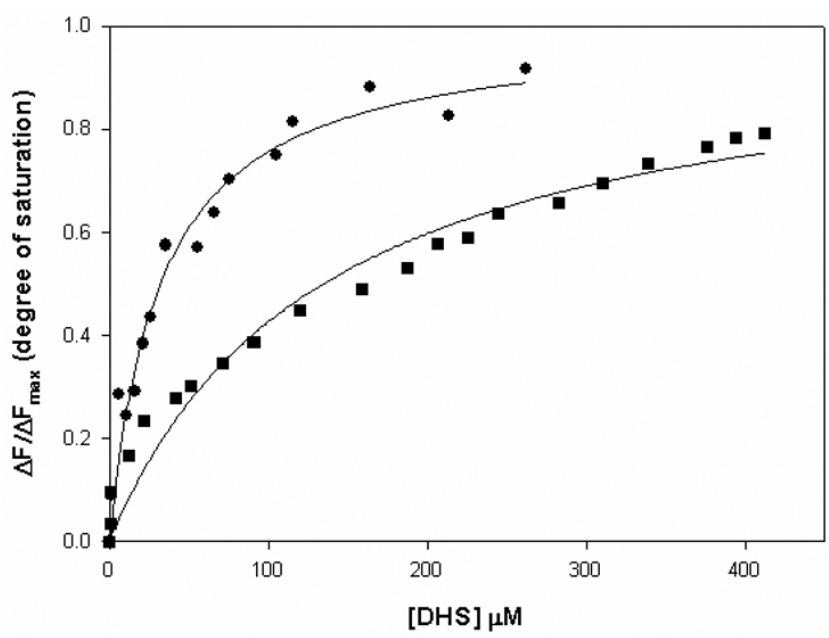

Figure 3

Spectroscopic measurements of intrinsic protein fluorescence for wild-type (black circles) and K69A (black squares) MtbSD upon DHS binding at varying concentrations.

(PDB). T. thermophilus SD structure was chosen as template for comparative homology modeling of MtbSD K69A and for SHK binding analysis because of larger primary sequence identity to MtbSD ( $30 \%$ identity and $13 \%$ strong similarity) as compared to A. aeolicus (24\% identity and 16\% strong similarity), and lower requirement for inclusion of gaps in primary sequence comparisons.

Analysis of the 3D structures of SDs in complex with $\mathrm{NADP}^{+}$and SHK suggest that Lys64 in T. termophilus [15] and Lys70 in A. aeolicus [23] interact with C3 of DHS and act as an acid-base catalytic group. Corresponding Lys residue is also suggested to be catalytically active in Staphylococcus epidermidis [24], Haemophilus influenzae [25] and Arabidopsis thaliana [26] SDs.

No significant changes were observed on protein's overall tertiary structure and in DHS/SHK binding site after energy minimization of the MtbSD K69A model. The RMSD deviation from template of only $0.55 \AA$, 99\% of amino acids within allowed regions of the Ramachandran's plots and PROCHECK parameters indicate that the K69A model satisfies all stereochemical requirements. Considering only substrate binding site amino acids, MtbSD K69A RMSD values reduced to $0.12 \AA$ when compared to template. Such minor structural variations indicate a strong conservation of the amino acid arrangement at the enzyme's substrate binding site, not only at their $\alpha$-carbon position but also in regard to their $\chi$ rotational angles, where only $M t b S D$ residue Ser20 (Ser16 on template) shows variation on its rotational

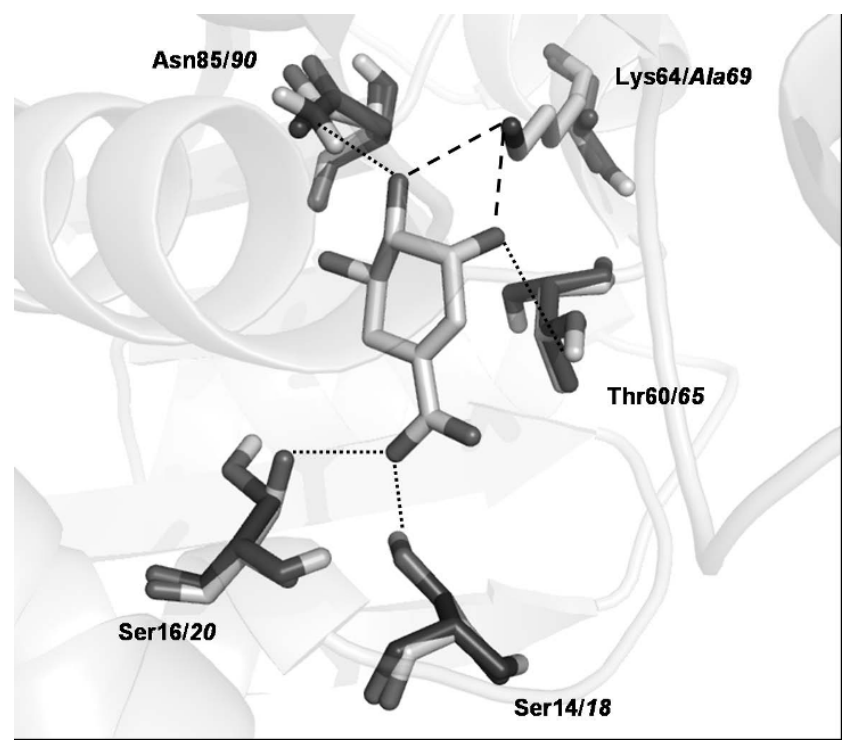

Figure 4

MtbSD K69A model superimposed on experimentally solved $T$. thermophilus SD structure. Amino acid side chains involved in SHK binding and SHK molecule are shown as sticks. T. thermophilus and MtbSD K69A amino acids are colored, respectively, in light gray (residue number in bold) and dark gray (residue number in italics). $\mathrm{H}$-bonds are shown as dotted lines; dashed lines represent $\mathrm{H}$-bonds between Lys64/69, missing in MtbSD K69A model.

angle $\chi_{1}$ (2.02 $\AA$ for $\mathrm{O} \gamma$ atom), although not affecting H-bonding to SHK. (Fig. 4).

\section{H-bonding pattern between MtbSD and SHK}

LIGPLOT analysis showed that the pattern of H-bonding to SHK is conserved in T. thermophilus SD and MtbSD K69A (Table 2). Gln243 residue in $M$. tuberculosis (corresponding to Gln235 in T. thermophilus SD) also makes a hydrophobic contact to C5 of SHK, as observed on template's structure. No significant amino acid

Table 2: Hydrogen bonding pattern of SD enzyme and SHK substrate. T. thermophilus values are shown in bold, MtbSD K69A corresponding values are shown in italics.

\begin{tabular}{|c|c|c|c|}
\hline SD residue & SD Atom & SHK atom & H bond distance $(\AA)$ \\
\hline Ser I 4/Ser 18 & $\mathbf{O \gamma}$ & O2 & $2.62 / 2.55$ \\
\hline Ser $16 /$ Ser20 & $\mathbf{O \gamma}$ & 02 & $2.72 / 2.73$ \\
\hline Thr60/Thr65 & $0 \gamma 1$ & 011 & $3.28 / 3.22$ \\
\hline Lys64/Lys69 & $\mathbf{N} \zeta$ & 011 & $2.80 /-$ \\
\hline Lys64/Lys69 & $\mathbf{N} \zeta$ & 012 & $3.10 /-$ \\
\hline Asn85/Asn90 & N $\delta 2$ & 012 & $3.15 / 2.93$ \\
\hline
\end{tabular}

Atom numbering as [24]*.

* O2 corresponds to carboxyl negative oxygen atom, OII to hydroxyl oxygen bound to $\mathrm{C} 3, \mathrm{O} / 2$ to hydroxyl oxygen bound to $\mathrm{C} 4$, and $\mathrm{O} 7$ to hydroxyl oxygen bound to $\mathrm{C} 5$, as depicted in Fig. I. 
rearrangements were observed for MtbSD K69A. Not surprisingly, the noticeable change is the loss of two $\mathrm{H}$ bonds with the SHK molecule in K69A mutant. It could be argued that the loss of two H-bonds in K69A MtbSD mutant would demonstrate the role of Lys69 in DHS/ SHK binding. The energies of hydrogen bonds have been variously estimated to be between 12 and $38 \mathrm{~kJ} \mathrm{~mol}^{-1}$ [22]. Spectrofluorimetric measurements showed a reduction of $3.5 \mathrm{~kJ} \mathrm{~mol}^{-1}$ in binding energy of DHS to K69A $\mathrm{M} t b \mathrm{SD}$ as compared to the wild-type enzyme.

\section{Conclusion}

Based on double isotope effects and pH-rate profiles, we have previously proposed that the chemical mechanism for MtbSD involves hydride transfer and solvent proton transfer in a concerted manner, and that an amino acid residue with an apparent $\mathrm{pK}_{\mathrm{a}}$ value of 8.9 is involved in catalysis [10]. Here we demonstrate that the MtbSD Lys69 is important for catalysis and is likely involved in stabilization of the developing negative charge at the hydride-accepting C-3 carbonyl oxygen of DHS for the forward reaction, acting as an acid-base catalytic group that donates a proton to DHS carbonyl group during reduction, playing a minor role, if any, in substrate binding.

In bacteria, four subclasses of SD have been identified, distinguished by their phylogeny and biochemical activity [27]. In agreement with our results, mutagenesis studies showed that the corresponding residues Lys67 in Haemophilus influenzae SD [25] and Lys385 in Arabidopsis thaliana dehydroquinate dehydratase-SD [26] play a critical role in catalysis and no role in substrate binding. Interestingly, site-directed mutagenesis of Escherichia coli YdiB (a bifunctional enzyme that catalyzes the reversible reductions of 3-dehydroquinate to quinate and 3dehydroshikimate to shikimate using as co-substrate either NADH or NADPH) has shown that the conserved Lys71 residue plays a primary role in substrate binding in the Michaelis complex and, although it contributes to some extent to transition state stabilization, plays no essential role in catalysis [28].

It should be pointed out that mechanistic analysis should always be a top priority for new enzyme-targeted drug programs since effective enzyme inhibitors take advantage of enzyme chemistry to achieve inhibition [29]. Accordingly, we hope that the results here presented will pave the way for the target-based rational design of novel effective antitubercular agents. The design of inhibitors of MtbSD enzyme activity may contemplate derivatization of $\mathrm{C}-3$ of DHS with functional groups that make strong interactions with the Lys69 side chain. It would appear to be unlikely that a mutation of Lys69 residue would be selected for to afford drug resistance because here is shown that this amino acid residue plays a critical role in MtbSD enzyme activity. However, an important additional point to be considered when selecting a particular enzyme as a successful drug target is to determine a flux control coefficient, which measures the sensitivity of flux to a change in enzyme concentration. An enzyme is likely a good target if the flux control coefficient is high, whereas it is less likely a good target if the flux control coefficient is close to zero (virtually all the activity must be eliminated by an inhibitor to have the desired chemotherapeutic effect). Bifunctional dehydroquinate dehydratase-shikimate dehydrogenases in plants are responsible for preferential routing of carbon to aromatic amino acid synthesis [30]. However, how shikimate dehydrogenase affects the shikimate pathway flux in M. tuberculosis is still unknown. Therefore, strategies for drug design contemplating derivatization of C-3 of DHS should also take into account whether shikimate dehydrogenase has a low flux control coefficient because mutation of Lys69 would be one way of gaining resistance to this enzyme's inhibitors.

\section{List of abbreviations used}

TB: tuberculosis; MDR: multi-drug resistant; XDR: extensively drug-resistant; MtbSD: shikimate dehydrogenase from $M$. tuberculosis; DHS: 3-dehydroshikimate; SHK: shikimate; $\mathrm{K}_{\mathrm{m}}$ : Michaelis-Menten constant; RMSD: root-mean square deviation; $\mathrm{k}_{\text {cat }}$ : catalytic constant; $\mathrm{K}_{\mathrm{d}}$ : equilibrium dissociation constant.

\section{Competing interests}

The authors declare that they have no competing interests.

\section{Author's contributions}

VSRJ performed most of the experiments and drafted the manuscript. $\mathrm{AB}$ performed the structural analysis and helped to write the manuscript. The study was conceived and coordinated by DSS and LAB, who have also helped to draft the manuscript. All authors read and approved the final version of the manuscript.

\section{Acknowledgements}

This work was supported by National Institute of Science and Technology on Tuberculosis (Decit/SCTIE/MS-MCT-CNPq-FNDCT-CAPES) and Millennium Initiative Program (CNPq), Brazil, to D.S.S. and L.A.B. D.S.S. and L.A.B. also acknowledge grants awarded by FINEP. D.S.S. (30405 I/I97506) and L.A.B. (520182/99-5) are research career awardees from the National Council for Scientific and Technological Development of Brazil (CNPq). We are grateful to Professor John W. Frost, Department of Chemistry of Michigan State University, for his generous gift of 3dehydroshikimate. 


\section{References}

I. Dye C, Scheele S, Dolin P, Pathania V and Raviglione MC: Globa burden of tuberculosis: estimated incidence, prevalence, and mortality by country. JAMA 1999, 282:677-686.

2. Basso LA and Blanchard JS: Resistance to antitubercular drugs. Adv Exp Med Biol 1998, 456: I I5-I44.

3. Dorman SE and Chaisson RE: From magic bullets back to the Magic Mountain: the rise of extensively drug-resistant tuberculosis. Nat Med 2007, 13:295-298.

4. Robertson JG: Mechanistic basis of enzyme-targeted drugs. Biochemistry 2005, 44:556I-557I.

5. Bentley R: The shikimate pathway - a metabolic tree with many branches. Crit Rev Biochem Mol Biol 1990, 25:307-384.

6. Ducati RG, Basso LA and Santos DS: Mycobacterial shikimate pathway enzymes as targets for drug design. Curr Drug Targets 2007, 8:423-435.

7. Parish $\mathrm{T}$ and Stoker NG: The common aromatic amino acid biosynthesis pathway is essential in Mycobacterium tuberculosis. Microbiology 2002, I48:3069-3077.

8. Magalhães MLB, Pereira CP, Basso LA and Santos DS: Cloning and expression of functional shikimate dehydrogenase (EC I.I.I.25) from Mycobacterium tuberculosis H37Rv. Prot Expr Purif 2002, 26:59-64.

9. Fonseca IO, Magalhães MLB, Oliveira JS, Silva RG, Mendes MA Palma MS, Santos DS and Basso LA: Functional shikimate dehydrogenase from Mycobacterium tuberculosis H37Rv: purification and characterization. Prot Expr Purif 2006 46:429-437.

10. Fonseca IO, Silva RG, Fernandes CL de Souza ON, Basso LA and Santos DS: Kinetic and chemical mechanisms of shikimate dehydrogenase from Mycobacterium tuberculosis. Arch Biochem Biophys 2007, 457:123-133.

II. Arcuri HA, Borges JC, Fonseca IO, Pereira JH, Neto JR, Basso LA Santos DS and de Azevedo WF Jr: Structural studies of shikimate 5-dehydrogenase from Mycobacterium tuberculosis. Proteins 2008, 72:720-730.

12. Rodrigues-Junior VS, Basso LA and Santos DS: Homogeneous recombinant Mycobacterium tuberculosis shikimate dehydrogenase production: an essential step towards target-based drug design. Int J Biol Macromol 2009, 45:200-205.

13. Laemmli UK: Cleavage of structural proteins during the assembly of the head of bacteriophage T4. Nature 1970 227:680-685.

14. Bradford MM, McRorie RA and Williams WL: $\mathbf{A}$ rapid and sensitive method for the quantification of microgram quantities of protein utilizing the principle of protein-dye binding. Anal Biochem 1976, 72:248-254

15. Bagautdinov B and Kunishima N: Crystal structures of shikimate dehydrogenase AroE from Thermus thermophilus HB8 and its cofactor and substrate complexes: insights into the enzymatic mechanism. J Mol Biol 2007, 373:424-438.

16. Šali A and Blundell TL: Comparative Protein Modelling by Satisfaction of Spatial Restraints. I Mol Biol I993, 234:779-8I 5 .

17. Martí-Renom MA, Stuart AC, Fiser A, Sánchez R, Melo F and Šali A Comparative Protein Structure Modeling of Genes and Genomes. Annu Rev Biophys Biomol Struct 2000, 29:29I-325.

18. Sali A and Overington JP: Derivation of rules for comparative protein modelling from a database of protein structure alignments - Modeling mutations and homologous proteins. Protein Science 1994, 3:1582-1596.

19. Shen MY and Šali A: Statistical potential for assessment and prediction of protein structures. Protein Science 2006, I 5:2507-2524.

20. Spoel van der $D$, Lindahl $E$, Hess B, Groenhof G, Mark AE and Berendsen HJC: GROMACS: fast, flexible, and free. J Comp Chem 2005, 26:170I-1718.

21. Wallace AC, Laskowski RA and Thornton JM: LIGPLOT: program to generate schematic diagrams of protein-ligand interactions. Prot Eng 1995, 8:127-134

22. Fersht A: Structure and Mechanism in Protein Science. W. H. Freeman and Company, New York; first 1999.

23. Gan J, Wu Y, Prabakaran P, Gu Y, Li Y, Andrykovitch M, Liu H, Gong $Y, Y$ an $H$ and Ji $X$ : Structural and biochemical analyses of shikimate dehydrogenase AroE from Aquiflex aeolicus: implications for the catalytic mechanism. Biochemistry 2007. 46:9513-9522

24. Han C, Hu T, Wu D, Qu S, Zhou J, Ding J, Shen X, Qu D and Jiang H: $X$-ray crystallographic and enzymatic analyses of shikimate dehydrogenases from Staphylococcus epidermidis. FEBS J 2009. 276: I 125-1139.
25. Singh S, Korolev S, Koroleva O, Zarembinski T, Collart F, Joachimiak $A$ and Christendat D: Crystal structure of a nove shikimate dehydrogenase from Haemophilus influenzae. J Biol Chem 2005, 280:1710I-I7I08.

26. Singh SA and Christendat D: Structure of Arabidopsis dehydroquinate dehydratase-shikimate dehydrogenase and implications for metabolic channeling in the shikimate pathway. Biochemistry 2006, 45:7787-7796.

27. Singh S, Stavrinides J Christendat D and Guttman DS: A phylogenomic analysis of the shikimate dehydrogenases reveals broadscale functional diversification and identifies one functionally distinct subclass. Mol Biol Evol 2008 , 25:2221-2232

28. Lindner HA, Nadeau G, Matte A, Michel G, Ménard R and Cygler M: Site-directed mutagenesis of the active site region in the quinate/shikimate 5-dehydrogenase YdiB of Escherichia coli. J Biol Chem 2005, 280:7162-7169.

29. Robertson JG: Enzymes as a special class of therapeutic target: clinical drugs and modes of action. Curr Opin Struct Biol 2007, I 7:674-679.

30. Moore B: Bifunctional and moonlighting enzymes: lighting the way to regulatory control. Trends Plant Sci 2004, 9:22 I-228.

Publish with BioMed Central and every scientist can read your work free of charge

"BioMed Central will be the most significant development for disseminating the results of biomedical research in our lifetime. "

Sir Paul Nurse, Cancer Research UK

Your research papers will be:

- available free of charge to the entire biomedical community

- peer reviewed and published immediately upon acceptance

- cited in PubMed and archived on PubMed Central

- yours - you keep the copyright

Submit your manuscript here:

http://www.biomedcentral.com/info/publishing_adv.asp
BioMedcentral 